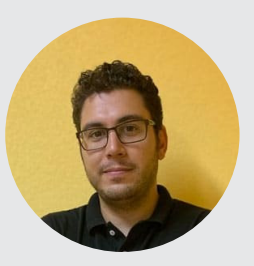

ALEXANDRE SOTELINO LOSADA

UNIVERSIDADE DE SANTIAGO DE COMPOSTELA

alexandre.sotelino@usc.es

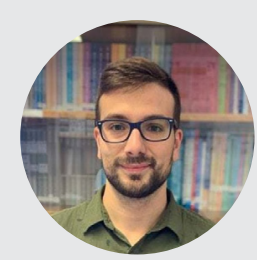

ÍGOR MELLA NÚÑEZ

UNIVERSIDADE DE SANTIAGO DE COMPOSTELA

igor.mella@usc.es

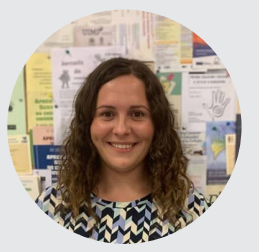

CRISTINA VARELA PORTELA

UNIVERSIDADE DE SANTIAGO DE COMPOSTELA

cristina.varela@usc.es

\section{Aprendizaje-servicio, TIC y conocimiento compartido para promover la reflexión en red}

\section{Service-learning, ICT and shared knowledge to promote reflection on the net}

Recibido: 23 de abril de 2020 / Aprobado: 23 de mayo de 2020

\section{Resumen}

El aprendizaje-servicio (ApS) ha surgido en los últimos años como una metodología innovadora en las aulas universitarias, atendiendo a criterios de transferencia y aprendizaje. Las evidencias en torno a su eficacia pedagógica son claras, apuntando a ganancias en términos académicos, cívico-sociales y profesionales. Asimismo, en tanto que se basa en la acción de los estudiantes en un marco comunitario, la reflexión sobre la propia experiencia se convierte en el principal catalizador para vincular servicio a la comunidad y aprendizaje curricular. Pero el avance que supone la sociedad de conocimiento plantea un reto epistemológico que invita a ajustar la inevitable presencialidad del ApS con tecnologías virtuales que promuevan la conexión y mejora de los procesos. Así pues, el presente artículo analiza el desafío de introducir recursos tecnológicos en los proyectos de aprendizaje-servicio universitarios, planteando como objetivo central vislumbrar algunas recomendaciones que puedan ser de utilidad en el desarrollo pragmático de esta metodología, a raíz de las posibilidades que traen consigo las tecnologías digitales como recurso para dar soporte a los procesos de reflexión educativa.

Palabras clave: aprendizaje-servicio; reflexión; tecnologías de la información y la comunicación; educación superior; sociedad del conocimiento.

\title{
Abstract
}

Service-learning (S-L) has emerged in recent years as an innovative methodology in university classrooms, attending to criteria of transference and learning. The evidences around its

\footnotetext{
1. Este trabajo se deriva del Proyecto de $1+D+i$ "Aprendizaje-servicio (ApS) y empleabilidad de los estudiantes universitarios en España: competencias para la inserción laboral", financiado por el Ministerio de Ciencia e Innovación, y fondos FEDER, como proyecto de I+D+i "Retos Investigación" del Programa Estatal de I+D+i Orientada a los Retos de la Sociedad (EDU2017-82629-R) - https://www.usc.gal/apsuni/apsuni-e/

a. Doctor en Ciencias de la Educación, GI-Esculca. Universidade de Santiago de Compostela (España). Para contactar al autor: alexandre.sotelino@usc.es

b. Doctor en Educación, GI-Esculca. Universidade de Santiago de Compostela (España). Para contactar al autor: igor.mella@usc.es

c. Doctora en Ciencias de la Educación, GI-Esculca. Universidade de Santiago de Compostela (España). Para contactar al autor: cristina.varela@ usc.es
} 
pedagogical efficacy are clear, focusing on gains in academic, civic, and professional terms. Likewise, insofar as it is based on student action in a community framework, reflection on one's own experience becomes the main catalyst to link community service and curricular learning. But the advance represented by the knowledge society poses an epistemological challenge that invites us to adjust the inevitable on-site component of S-L with virtual technologies that promote the connection and improvement of processes. Thus, this article analyses the challenge of introducing technological resources in university service-learning projects, proposing as a central objective to glimpse patterns that may be useful in the pragmatic development of this methodology, resulting of the possibilities of digital technologies as a resource to support educational reflection processes.

Keywords: service-learning; reflection; information and communication technologies; higher education; knowledge society.

\section{Introducción}

Actualmente, la educación superior se sitúa en uno de los momentos de transición más notorios de toda su historia, dada la transformación que están experimentando sus dos tradicionales funciones (docencia e investigación), así como la aparición de una tercera función que impregna de responsabilidad y compromiso social a todas las tareas que son propias de la actividad universitaria. La coyuntura social en la que se mueven las instituciones universitarias hoy en día ha causado una vasta transformación de los procesos que en ellas tienen lugar, a la vez que les reclaman un fuerte protagonismo como vectores de transformación y avance de la sociedad. No en vano, la Universidad desempeña un rol principal en el asentamiento y desarrollo de la que vino en denominarse "sociedad del conocimiento" (Santos Rego, 2016).

En este contexto, donde el conocimiento se convierte en el principal eje de avance y desarrollo social, la Universidad ha procedido a una remodelación de los procesos de enseñanza-aprendizaje, adoptando un nuevo paradigma formativo donde lo que importa es el trabajo de los estudiantes y su autonomía en la construcción de conocimiento. Es por ello, que las Universidades e Institutos de Educación Superior deben repensar sus modelos basados en la transmisión unidireccional de información en los que el docente es quién tiene el conocimiento en su poder, para entender que la formación del alumnado debe producirse en diálogo con la comunidad (Sotelino, Santos Rego, y Lorenzo, 2016). Surgen así metodologías de corte innovador que tratan de dar respuesta al nuevo modelo de formación universitaria, como es el caso de la que nos ocupa en este artículo, el aprendizaje-servicio, en la que se combina el aprendizaje académico con la acción del individuo en un entorno de servicio comunitario.

Así pues, los proyectos de aprendizajeservicio promueven procesos de aprendizaje centrados en el alumnado y en su acción (Gargallo, Suárez, Garfella, y Fernández March, 2011), todo ello en un marco de relaciones con la comunidad en el que se espera una optimización de los resultados cognitivos y sociales (Mella, Santos Rego, y Malheiro, 2015; Naval y Arbués, 2018). Como iniciativa educativa que se basa en la experiencia de los estudiantes, requiere de procesos de reflexión continuados y guiados que permitan dotar de sentido a la propia acción y, en concreto, establecer vínculos críticos y fundamentados entre las actividades de servicio desarrolladas y los contenidos disciplinares de referencia.

Pero el aprendizaje-servicio, aun enfren- 
tando el fenómeno educativo de un modo ciertamente integral, ha de buscar vías de desarrollo en conexión con otras iniciativas y realidades que atañen a la Universidad en el siglo XXI. Tal es el caso de la introducción y expansión de las tecnologías de la información y la comunicación (TIC) para dar apoyo a los procesos educativos. No debemos olvidar que, en el caso de la Universidad europea, se reconoce que uno de los principales desafíos pedagógicos que se derivan de la implantación del Espacio Europeo de Educación Superior (EEES) es la necesidad de fomentar la acogida $y$ utilización de las TIC en la docencia, partiendo siempre de una adecuada dotación de recursos a las Universidades (Calderón y Escalera, 2008).

Incluso, en palabras de Bringle y Hatcher (2007), el aprendizaje-servicio y las TIC se configuran como las dos innovaciones que se han adentrado con mayor fuerza en la Universidad estadounidense, amén de las que mayores posibilidades de transformación parecen aportar. Este argumento da buena muestra del interés que tiene analizar en el presente trabajo las posibilidades de conexión que se establecen entre estas dos realidades.

A raíz de lo dicho hasta ahora, el objetivo último del estudio no es otro que el de analizar la posible integración de las TIC en los proyectos de aprendizaje-servicio, prestando especial atención a las vías que así se abren para impulsar procesos de reflexión crítica que se basen en la colaboración y en la conexión, ya no solo entre estudiantes, sino en un plano, incluso, global.

Por tanto, y para la consecución de esta finalidad, haremos un desarrollo argumental que pasa por justificar la importancia y potencialidad del aprendizaje-servicio en el ámbito universitario, para posteriormente hacer un desarrollo teórico donde se detallen las posibilidades que ofrecen actualmente las TIC para fomentar la reflexión en este tipo de aprendizaje experiencial. Toda la línea discursiva estará basada en una metodología cualitativa de exhaustiva revisión bibliográfica sumada a la experiencia en proyectos de investigación competitiva, y el desarrollo y asesoramiento de proyectos de ApS en diferentes Universidades por todo el mundo (Nieto, 2010; Pérez-Serrano, 2011).

\section{El aprendizaje-servicio en la educación superior: ejes para un desarrollo sostenible}

El papel de las Universidades ha sido modificado en los últimos años $\mathrm{y}$, en los albores del segundo cuarto del siglo XXI, los grandes retos educativos pasarán por la combinación efectiva de cuatro factores: crear y transmitir aprendizajes más pragmáticos, promover una mayor responsabilidad ciudadana y ambiental, contribuir y estudiar el desarrollo tecnológico, y guiar los avan/ ces científicos e industriales desde una perspectiva de justicia social (Gallardo, De Castro, y Saiz, 2020; Gotor, 2015; Monge, 2003). Es por ello, que muchas convocatorias de programas de investigación están planteando estas líneas como marco referente. Urgen, consecuentemente, iniciativas encaminadas a establecer recomendaciones sobre el modo en que las metodologías y recursos de aula pueden adaptarse a aquello que en el ámbito científico ya es una realidad.

Al mismo tiempo, las universidades deben tomar como propia la misión de gestionar sus actividades al servicio del entorno social más próximo, alejándose de la tradicional visión cual torre de marfil. Hoy más que nunca, tienen que establecer conexiones directas. Los vínculos entre Universidad y sociedad se hacen necesarios para garantizar una investigación de utilidad, toda vez que la realidad inmediata es la que ha de motivar dicha investigación y a la que se deben transferir los resultados oportunos. Ahora bien, la Academia ha tenido la mira puesta en sus dos tradicionales funciones, a saber, la docencia y la investigación, descuidando en algunas 
ocasiones su tercera misión (propósito de transferencia), esto es, la responsabilidad social. Llega ahora el momento de reconciliar estos tres pilares que sostienen la educación superior, buscando vínculos que converjan en procesos únicos.

En este sentido, el aprendizaje-servicio puede ser de gran utilidad para fortalecer la dimensión social de la educación superior por medio de una de sus actividades principales, la docencia, y con su colectivo más numeroso, los estudiantes. Una definición clara es la que nos ofrecen Puig, Batlle, Bosch, y Palos (2007, p. 20), al presentarla como una "propuesta educativa que combina procesos de aprendizaje y de servicio a la comunidad en un solo proyecto bien articulado en el que los participantes se forman al trabajar sobre necesidades reales del entorno con el objetivo de mejorarlo".

Por tanto, esta metodología permite, e incluso exige, un alto grado de participación por parte de todos los agentes que en ella se implican. Al respecto, es destacable el caso de los estudiantes, en tanto que las diferentes actividades parten de la negociación y el consenso, lo que da lugar a un vínculo emocional con el proyecto que amplía la motivación intrínseca en su desarrollo e implementación. Esta idea conlleva también un ejercicio de auténtica democracia, entendida esta como la participación de la ciudadanía en los procesos que importan en el nivel social $y$ comunitario, pues no debemos olvidar que el aprendizaje-servicio supone oportunidades para que los estudiantes de diferentes niveles educativos se involucren en actividades y acciones con fuerte calado social (Sotelino, Mella, y Rodríguez Fernández, 2019).

\section{El ApS permite hacer frente a las} recomendaciones educativas que se derivan de la creación del EEES, sobre todo en lo que se refiere al fortalecimiento de la dimensión social del aprendizaje y la promoción de procesos centrados en el trabajo del alumnado, aminorando la relevancia de la simple transmisión de información por parte del docente. Ahora bien, no resulta ni mucho menos suficiente que la introducción y expansión del ApS en la universidad dependa de la motivación del profesorado, sino que las instituciones deben ofrecer el respaldo y apoyo necesario, sobre todo al amparo del fortalecimiento de los procesos de enseñanzaaprendizaje y de las políticas de compromiso social (Lorenzo, Mella, García y Varela, 2017).

Consecuentemente, dado su claro potencial pedagógico, algunas Universidades están dando pasos en la institucionalización de esta metodología, disponiendo los recursos y el soporte necesario para garantizar una expansión sostenible y perdurable (Martínez-Usarralde, Gil-Salom, y MacíasMendoza, 2019; Santos Rego y Lorenzo, 2018). Pero este desarrollo y consolidación de la metodología debe responder a criterios de calidad en las experiencias, y no solo ser el resultado de una tendencia o moda pasajera. En esta vía se expresaban Santos Rego, Lorenzo y Sotelino (2018, pp. 7-8):

[S]i somos capaces de proceder con un esquema de institucionalización efectivamente gradual, cuidando la calidad de las dinámicas de aprendizaje-servicio en el seno de las universidades, estaremos contribuyendo a motivar al profesorado con predisposición favorable a poner en marcha estos proyectos, al contar con un soporte que avala y contribuye a su implantación. Lo que, en última instancia, se debe traducir en una mejora de la formación del alumnado.

En definitiva, de lo que se trata en el aprendizaje-servicio es de que el alumnado aprenda más contextualizadamente, para lo que se ha de atender a criterios profesionales y cívicos (Naval y Arbués, 2016). No obstante, conviene tener presente que pueden existir situaciones concretas donde no se adapte a las exigencias curriculares (Vázquez Verdera, 2015). Por ello, es importante indicar que su desarrollo efectivo ha de ser claramente sostenible y bajo 
criterios de calidad, siendo uno de los principales el instalar procesos rigurosos de reflexión que permitan crear aprendizaje y sentido (académico, social y profesional) en torno a la experiencia de servicio.

\section{Aunar aprendizaje y servicio: la reflexión como nexo}

El pensamiento crítico que se desarrolla a lo largo de toda experiencia de aprendizajeservicio permite que el alumnado interiorice aquellas actuaciones que ha realizado $y$ establezca las principales conexiones entre el currículum y la actividad desarrollada en la comunidad (Martínez-Odría, 2007). Es por ello por lo que entendemos la reflexión como elemento básico de esta metodología y como principal criterio de calidad. En consonancia con Santos Rego et al. (2015), podemos decir que la utilidad y calidad de un proyecto de aprendizaje-servicio se correlacionan con el proceso de reflexión en torno al mismo.

Si bien debemos, en primera instancia, plantearnos qué es lo que entendemos por reflexión, término que Escofet y Rubio (2017) delimitan como uno de los elementos en torno a los que se configura el actual cambio educativo experimentado en la Universidad, el cual deja atrás la recepción pasiva y acrítica de información y apunta hacia un modelo pedagógico centrado en el aprendizaje autónomo y activo de los estudiantes.

No nos referimos a una reflexión puntual y de bajo perfil, como bien apuntan Páez y Puig (2013), sino a aquella que es organizada intencionalmente por alumnado y profesorado. En esta línea, Eyler, Giles y Schmiede (1996) presentan los principios que debe seguir un proceso reflexivo para ser de calidad: ser continuado (antes, durante y después de la experiencia); conectado con los objetivos intelectuales y académicos de los estudiantes; desafiante, en tanto que implica la resolución de problemas de forma crítica; y, contextualizado, ajustándose a cada proyecto.
Por su parte, Bringle y Hatcher (1999) proponen que se deben tener en cuenta los siguientes aspectos: ha de unir claramente el servicio con el contenido de la asignatura y los objetivos de aprendizaje; las expectativas y criterios para la evaluación deben estar delimitadas en términos de descripción; debe ser regular y continuado; debe ofrecer feedback por parte del profesorado; e incluir oportunidades de exploración, clarificación y alteración de los propios valores para el alumnado.

Tomando en consideración la transversalidad y continuidad del proyecto, Eyler (2002) nos presenta las principales características de los procesos de reflexión en función de los tres momentos en los que ha de desarrollarse: antes, durante y después del servicio. Así, la reflexión antes del servicio se centra en el análisis de las percepciones del alumnado en torno a la comunidad, de cara a elevar su nivel de conciencia para identificar los principales conflictos entre las nuevas experiencias y las antiguas asunciones. Si hablamos de la reflexión durante el servicio, cabe señalar que permite a los estudiantes enfrentarse a esas discrepancias que proceden de las asunciones previas, tomando ya en consideración los marcos de referencia actuales. $Y$, por último, la reflexión una vez termina el servicio a la comunidad, momento en el que se trata de consolidar el aprendizaje, examinar la comprensión de la asignatura e identificar aquellos problemas que todavía no se han solucionado.

En cuanto a los formatos de reflexión, es necesario hacerse eco de la gran variedad de procedimientos existentes, ya que puede ser escrita, oral o ambas; individual, colaborativa, o ambas; planificada en el tiempo o informal y casual; puede suponer feedback de numerosos colectivos; y puede ejecutarse en numerosos soportes y actividades (Bringle y Clayton, 2012). Al respecto, Eyler et al. (1996) tratan de categorizar las actividades de reflexión en cuatro tipos: 
a Leer. Hablamos de lecturas que ofrecen teorías y modelos racionales y lógicos que ayudan al alumnado a crear sentido de sus experiencias de servicio.

Escribir. Entendiendo la escritura bien como un procedimiento para integrar las propias experiencias con modelos y enfoques teóricos, o bien como una herramienta con la que proponer ideas y proyectos derivados de su aprendizaje.

Hacer. Este tipo de actividades se dirigen a estilos de aprendizaje más activos, donde se aprende a partir de la inmersión en un proyecto concreto.

Decir. Las presentaciones orales pueden, también, suponer cierto impacto o cambio en el alumnado, pues permiten compartir las diferentes experiencias.

No es arriesgado señalar que la clave para optimizar el potencial del aprendizajeservicio es el favorecer aquellos procesos de reflexión realmente efectivos. De acuerdo con Ash, Clayton y Atkinson (2005), entendemos que este es el elemento que permite al alumnado dar cuenta de cómo ha adquirido los diferentes aprendizajes, convirtiéndose, de este modo, en un mecanismo óptimo para evaluarlos.

\section{Las TIC como herramienta para la reflexión en el ApS}

En el actual contexto, donde la Universidad se ve afectada por los retos que le formulan una sociedad y una economía basadas en el conocimiento, la irrupción de las tecnologías de la información y la comunicación se erige como uno de los principales factores a tomar en consideración. Para Välimaa y Hoffman (2008), las TIC afectan a la Universidad en un doble sentido: por un lado, como institución que se encarga, aunque no exclusivamente, de producir innovación tecnológica y, por otro, como usuaria y beneficiaria de dichas tecnologías. La vertiente que interesa en el presente artículo es la segunda, pues el uso educativo de las TIC ha supuesto una remodelación de los procesos de enseñanza-aprendizaje, con edificantes consecuencias en el rol que desempeñan tanto el profesorado como los alumnos.

Pero la importancia otorgada a las TIC en el nuevo modelo universitario no es injustificada, y autores como Villa (2006) la evidencian en las posibilidades que generan en cuanto a: atención a necesidades específicas e individuales; fomento de estilos de aprendizaje diferenciados; promoción de diferentes capacidades cognitivas, motivaciones y conocimientos previos del alumnado; estímulo de la interacción controlada y feedback; o fomento de procesos de aprendizaje autónomo, activo y significativo.

Lo que podemos concluir, a la luz de lo expuesto hasta aquí, y con más razón en la situación de riesgo en la que nos hemos visto sometidos en el año 2020, es la aparición de un nuevo modelo formativo en el que las TIC se convierten en la herramienta que da apoyo a los procesos de enseñanza-aprendizaje. Hablamos del aprendizaje electrónico o e-learning, que se define como "la utilización de las nuevas tecnologías multimediales y de Internet, para mejorar la calidad del aprendizaje facilitando el acceso a recursos y servicios, así como los intercambios y la colaboración a distancia" (Comisión de las Comunidades Europeas, 2001, p. 2). Es oportuno reparar aquí en que el e-learning no refiere únicamente a procesos educativos en línea o totalmente virtuales, sino a cualquier actividad pedagógica donde se hace uso de las TIC para mejorar el aprendizaje y establecer constructivas conexiones e interacciones.

Ahora bien, no debemos entender que la introducción de las TIC se desvincula de cualquier otro tipo de innovación educativa, sino que lo provechoso es que se produzca en concomitancia con otras iniciativas de 
renovación. Por eso, nos disponemos a continuación a analizar las posibilidades de combinación que se presentan entre el uso de tecnologías digitales y el AprendizajeServicio en el nuevo modelo de Universidad.

El vínculo entre ambas realidades se ha convertido en objeto de análisis en los últimos años (García-Gutiérrez y Ruiz-Corbella, 2020; Santos Rego, Mella, y Sotelino, 2020), si bien, a priori, pueden aparentar iniciativas de difícil combinación. Lo que aquí defendemos es una complementación recíproca entre las TIC y el Aprendizaje-Servicio: es decir, por un lado, el ApS permite dotar de significación comunitaria y cívica a los procesos educativos ciertamente impersonales que se articulan en torno a las TIC; y, por otro, las tecnologías facilitan que en el Aprendizaje-Servicio se articulen dinámicas de reflexión compartidas y conectadas, así como de difusión de la experiencia.

Así pues, surge una nueva modalidad de ApS cuando parte o el total del proceso se ve mediado por tecnologías digitales: el Aprendizaje-Servicio electrónico (e-ApS). Dentro de esta modalidad se pueden reconocer diferentes tipos en función del grado de virtualidad que se introduzca tanto en el propio proceso formativo e instructivo como en el servicio a la comunidad (Waldner, McGorry y Widener, 2012). Incluso, podemos hablar de variantes de Aprendizaje-Servicio totalmente virtual, donde el ApS permite introducir un componente comunitario y cívico en procesos de formación en línea, como es el caso de la Universidad Nacional de Educación a Distancia (UNED) de España (GarcíaGutiérrez, Ruiz-Corbella y del Pozo, 2017).

Pero lo que a nosotros nos interesa son las posibilidades que abren las TIC para apoyar determinadas actividades que se desarrollan en los proyectos de ApS (Tabla 1), en concreto la reflexión. Lo que nos lleva a pensar, principalmente, en iniciativas de Aprendizaje-Servicio tradicional donde las tecnologías son utilizadas como herramienta para dar apoyo e incrementar el intercambio y las interrelaciones que han de definir a este tipo de experiencias (Escofet, 2020).

Centramos ya nuestro análisis en las posibilidades que traen consigo las TIC para impulsar los procesos de reflexión que, como ya vimos en el anterior epígrafe, se sitúan como elemento nuclear en el ApS, pues permite vincular el servicio a la comunidad con el aprendizaje académico, a la vez que articula un proceso de aprendizaje significativo que se aleja de la asunción acrítica de principios y teorías. Cabe recordar aquí la apreciación de Waldner et al. (2012), para quienes un modelo óptimo de Aprendizaje-Servicio es aquel en el que tanto los procesos formativos como el servicio tienen lugar presencialmente, haciendo uso de las TIC para apoyar procesos de reflexión en los que la interacción con los compañeros

Tabla1. Actividades de un proyecto de ApS y tecnologías

\begin{tabular}{|c|c|}
\hline TAREA & EJEMPLO DE TECNOLOGía \\
\hline Participación del socio comunitario & Correo electrónico \\
Aprendizaje & Plataforma virtual de aprendizaje \\
Servicio & Crear una página web \\
Reflexión & Foro en línea \\
Evaluación del programa & Cuestionario en línea \\
\hline
\end{tabular}

Fuente: Escofet (2020) 
permite enriquecer el proceso.

Es más, cada experiencia individual en ApS, incluso en el marco de un mismo proyecto, es totalmente diferente, debido a la propia heterogeneidad del alumnado y de sus marcos contextuales. Por ello, la reflexión en entornos virtuales, como puede ser el caso de un foro en línea, abre posibilidades de compartir y conectar aprendizajes enormemente diversos (Guthrie y McCracken, 2014). Son numerosas las tecnologías que permitirán dar apoyo a los procesos de reflexión, entre las que podemos destacar la creación de blogs, las narrativas digitales, los foros virtuales o el uso de grupos de WhatsApp o de otras redes sociales (Escofet, 2020).

Ahora bien, el tipo de soporte electrónico en el que se apoye la reflexión dependerá de la modalidad reflexiva del proyecto en sí. Este es el argumento de Salam, Awang, Abang, y Farooq (2019), quienes sugieren diferentes tecnologías para cada subtipo de actividad de reflexión:

a Reflexión escrita. Puede hacerse uso de portafolios electrónicos, blogs o redes sociales como Twitter.

b Reflexión oral. En este caso se apoyaría en la utilización de todo tipo de dispositivos y software de grabación audiovisual.

Reflexión colaborativa. A fin de establecer redes y conexiones entre estudiantes, podría hacerse uso de foros de discusión virtuales.

No podemos pasar por alto las posibilidades de la reflexión electrónica para fomentar procesos formativos de tipo conectivo, pues lo que permite es una puesta en común directa de aprendizajes y experiencias heterogéneas que permiten crear significados propios e individuales en un marco de diálogo en torno a la diferencia. Las discusiones entre estudiantes en entornos virtuales permiten un intercambio de ideas enriquecedor, basándose en la diversidad de opiniones y argumentos. El profesorado es quien debe encargarse de guiarlas para que supongan reflexiones sobre las relaciones que existen entre las actividades de servicio a la comunidad y los contenidos de la asignatura, ya que no hemos de olvidar la naturaleza curricular del AprendizajeServicio (Guthrie y McCracken, 2014).

Asimismo, uno de los beneficios más destacados de que la reflexión haga uso de tecnologías digitales es la posibilidad de trascender el contexto del aula, de la institución e, incluso, el de la comunidad local o nacional. Así lo sugieren Bringle y Clayton (2020) al proponer las narrativas digitales como medio para fomentar la reflexión crítica, en tanto que permiten que el alumnado comparta y narre sus historias a través de diferentes soportes virtuales. Lo que resuelven es que este soporte reflexivo cuenta con tal nivel de proyección que permite contactar con individuos con los que de otro modo nunca se podría establecer comunicación, lo que trae consigo numerosas posibilidades de desarrollo $y$ aprendizaje cívico, sin olvidar el vínculo estrecho que ha de existir entre las historias digitales y los objetivos académicos. Cabe valorar aquí las oportunidades de interacción y colaboración entre agentes con objetivos compartidos, como pueden ser, por ejemplo, proyectos de ApS similares pero radicados en contextos geográficos distantes, lo que terminaría por enriquecer la calidad en la reflexión desarrollada.

Queremos detenernos en este punto sobre el que posiblemente sea el instrumento de reflexión más utilizado en el AprendizajeServicio: el portafolios (Santos Rego et al., 2015). Este instrumento se trata de una colección de documentos y otro tipo de materiales cuyo fin último es el de ofrecer evidencias de las competencias y los logros de los estudiantes, tomando siempre en consideración los objetivos de aprendizaje y el proceso por el que se han ido adquiriendo (Eyler et al., 1996). De su propia definición se desprenden las posibilidades que tiene el estructurar esta construcción reflexiva y 
personal en tecnologías digitales.

Hablamos, pues, del portafolios electrónico o digital (e-portafolio), donde la principal diferencia con respecto al modelo tradicional reside en las posibilidades de interacción que genera, la multimodalidad en el discurso y la creación de una comunidad de aprendizaje en la que se establecen conexiones de colaboración y se comparte el trabajo realizado (González y Montmany, 2019). Si atendemos a su definición, presentamos la que nos ofrecen Kunnari y Laurikainen (2017, p. 7):

Son espacios de trabajo y aprendizaje digital propiedad del alumno, para recolectar, crear, compartir, colaborar y reflexionar sobre el aprendizaje y las competencias, así como para recibir y recopilar las evaluaciones. Son plataformas para que los estudiantes se comprometan en su desarrollo personal y profesional, así como para que interactúen en comunidades de aprendizaje y con los diferentes agentes implicados en el proceso de aprendizaje.

En definitiva, lo que queremos transmitir aquí es la optimización de los procesos de reflexión que se deriva del uso de entornos virtuales para la creación de un portafolios personal. De este modo, la construcción personal se produce en torno a dinámicas de cooperación con los compañeros e, incluso, con otros agentes, lo que conlleva positivas posibilidades de retroalimentación.

Pero no debemos olvidar los retos y las dificultades que entraña el uso de las TIC en cualquier iniciativa educativa, realidad a la que no se escapan las actividades de reflexión en el ApS. El más destacado, tal y como nos indican Salam et al. (2019), es la dificultad que entraña para el profesorado reconocer los procesos de reflexión colaborativa que subyacen y que, en realidad, son el leit motiv para introducir componentes de virtualidad en los proyectos de ApS. Como indican los autores, no es sencillo para los docentes monitorizar y guiar los procesos de reflexión colaborativa y el intercambio que se produce cuando se hace uso de estrategias como las discusiones virtuales o los portafolios electrónicos, debido, especialmente, al elevado número de estudiantes con el que se cuenta en las aulas universitarias. Precisamente por eso, un proyecto de ApS electrónico demanda al profesorado un alto grado de experticia docente en esta metodología, pues, además del dominio de los contenidos, requiere una capacitación docente y didáctica adecuada (sobre todo para guiar los procesos de reflexión cooperativa), así como conocimientos tecnológicos ajustados al tipo de herramientas de las que se haga uso (Waldner et al., 2012).

\section{Conclusiones}

En un mundo claramente globalizado, donde los procesos de comunicación se han visto totalmente transformados y ampliados como consecuencia del avance tecnológico, las aulas universitarias no pueden permanecer ajenas a este nuevo escenario. Esto no quiere decir que todos los procesos tengan que producirse en entornos virtuales, puesto que negaríamos que la experimentación y la presencialidad, donde se producen enriquecedoras interacciones, tienen un peso sustancial en la formación de profesionales. Pero, las metodologías de las que se venía haciendo uso, e incluso aquellas de corte más innovador, como es el caso del Aprendizaje-Servicio, deberán empezar a indagar vías de inclusión de las TIC en su desarrollo, para no distanciarse de las dinámicas relacionales de las nuevas generaciones de estudiantes universitarios. Así, tendremos que trazar espacios donde el uso de medios virtuales pueda suponer una optimización de los procesos, y en última instancia redunden en la mejora de la adquisición de aprendizajes. En el caso del Aprendizaje-Servicio, el potencial de las TIC al respecto se sitúa, principalmente, en las posibilidades que genera de mayor conexión de un aprendizaje que, per se, surge de procesos colaborativos. Por tanto, permiten instalar iniciativas de reflexión crítica en las que el aprendizaje se 
construye en un entorno de conocimientos compartidos, a partir de las experiencias individuales que cada estudiante ha vivido en la comunidad.

Sin olvidar las posibilidades que abren para almacenar y recopilar evidencias y diferentes materiales que se derivan de la propia experiencia de aprendizaje, las TIC se convierten en el soporte en el que el alumno puede alojar gran cantidad de información derivada de su experiencia, pudiendo así organizarla en un entorno multimedial donde las posibilidades son, indudablemente, mayores a las que nos ofrecen otros recursos de tipo tradicional. Es el caso de los portafolios electrónicos, donde los estudiantes pueden ir estructurando todo tipo de evidencias y pruebas (textos, vídeos, páginas webs, audios, etc.) que contribuyan a potenciar una mirada analítica y reflexiva sobre la participación en el proyecto.

Por tanto, el uso de soportes virtuales en el aprendizaje-servicio contribuye innegablemente a abrir vías de desarrollo y expansión en los procesos de reflexión tradicionales. Instrumentos como pueden ser los foros virtuales abren canales de comunicación constante donde los estudiantes pueden reestructurar sus propias experiencias. Se establecen así nuevos cauces para un diálogo en el que los dilemas que se derivan de las experiencias de servicio se trabajan en un marco de colaboración bajo la supervisión del docente.

\section{Referencias}

Ash, S. L., Clayton, P. H., y Atkinson, M. P. (2005). Integrating reflection and assessment to capture and improve student learning. Michigan Journal of Community Service Learning, 11(2), 49-60.

Bringle, R. G., y Clayton, P. H. (2012). Civic education through service learning: what, how, and why? En L. Mcllrath, A. Lyons, y R. Munck (Eds.), Higher educa- tion and civic engagement. Comparative perspectives (pp. 101-124). Nueva York, NY: Palgrave Macmillan.

Bringle, R. G., y Clayton, P. H. (2020). Integrating service learning and digital technologies: examining the challenge and the promise. Revista Iberoamericana de Educación a Distancia, 23(1), 43-65. doi: 10.5944/ried.23.1.25386

Bringle, R. G., y Hatcher, J. A. (1999). Reflection in service learning: making meaning of experience. Educational Horizons, 77(4), 179-185.

Bringle, R. G., y Hatcher, J. A. (2007). Civic engagement and service learning: implications for higher education in America and South Africa. Education as Change, 11(3), 79-89. doi: 10.1080/16823200 709487181

Calderón, C., y Escalera, G. (2008). La evaluación de la docencia ante el reto de Espacio Europeo de Educación Superior (EEES). Educación XX1, 11, 237-256. doi: 10.5944/educxx1.11.0.316

Comisión de las Comunidades Europeas. (2001). Comunicación de la Comisión al Consejo y al Parlamento Europeo. Plan de acción elearning. Concebir la educación del futuro. Recuperado de http://eur-lex.europa.eu/legal-cont en t / E S / TXT/PD F / ? u r i = CE L EX:52001DC0172\&from=ES

Escofet, A. (2020). Aprendizaje-servicio y tecnologías digitales: ¿una relación posible? Revista Iberoamericana de Educación a Distancia, 23(1), 169-182. doi: 10.5944/ried.23.1.24680

Escofet, A., y Rubio, L. (2017). El valor de la reflexión en el aprendizaje-servicio. En L. Rubio y A. Escofet (Coords.), Aprendizaje-servicio (ApS): claves para su desarrollo en la universidad. Barcelona: Octaedro / ICE-UB.

Eyler, J. (2002). Reflection: Linking service and learning - linking students and communities. Journal of Social Issues, 58(3), 517-534. doi: 10.1111/1540-4560.00274 
Eyler, J., Giles, D. E., y Schmiede, A. (1996). A practitioner's guide to reflection in service-learning: student voices \& reflections. Nashville, TN: Vanderbilt University.

Gallardo, I. M., De Castro, A., y Saiz, H. (2020). Interacción y uso de tecnologías en los procesos de enseñanza y aprendizaje. Educatio siglo XXI: Revista de la Facultad de Educación, 38(1), 119-137.

García-Gutiérrez, J., Ruiz-Corbella, M., y del Pozo, A. (2017). Developing civic engagement in distance education: $A$ case study of virtual service-learning (vSL) programme in Spain. Open Praxis, 9(2), 235-244. doi: 10.5944/openpraxis. $9-2-578$

García-Gutiérrez, J., y Ruiz-Corbella, M. (2020). Aprendizaje-servicio y tecnologías digitales: un desafío para los espacios virtuales de aprendizaje. Revista Iberoamericana de Educación a Distancia, 23(1), 31-42. doi:10.5944/ ried. 23.1.25390

Gargallo, B., Suárez, J., Garfella, P. R., y Fernández March, A. (2011). El cuestionario CEMEDEPU. Un instrumento para la evaluación de la metodología docente y evaluativa de los profesores universitarios. Estudios Sobre Educación, 21, 9-40.

González, V., y Montmany, B. (2019). Iniciarse en el ámbito de los portafolios digitales. En J. T. Pujolà (Ed.), El portafolio digital en la docencia universitaria (pp. 11-26). Barcelona: Octaedro.

Gotor, V. M. (2015). Los desafíos de la universidad en el siglo XXI. En T. Luque (Ed.), Horizonte 2031: la Universidad de Granada ante su $V$ Centenario: reflexiones sobre el futuro de la universidad (pp 168-173). Granada: Editorial Universidad de Granada

Guthrie, K. L., y McCracken, H. (2014). Reflection: the importance of making meaning in e-service-learning courses. Journal of Computing in Higher Education, 26, 238-252. doi: 10.1007/s 12528-014-9087-9
Kunnari, I., y Laurikainen, M. (Eds.). (2017). Collection of engaging practices in eportfolio process - Publication of the empowering eportfolio process project. Hämeenlinna: Häme University of Applied Sciences.

Lorenzo, M., Mella, I., García, J., y Varela, C. (2017). Investigar para institucionalizar el aprendizaje servicio en la universidad española. RIDAS. Revista iberoamericana de aprendizaje servicio: Solidaridad, ciudadanía y educación, 3, 118-130.

Martínez-Odría, A. (2007). Service-learning o aprendizaje-servicio. La apertura de la escuela a la comunidad local como propuesta de educación para la ciudadanía. Bordón, 59(4), 627-640.

Martínez-Usarralde, M. J., Gil-Salom, D., y Macías-Mendoza, D. (2019). Revisión sistemática de Responsabilidad Social Universitaria y Aprendizaje Servicio. Análisis para su institucionalización. Revista Mexicana de Investigación Educativa, 24(80), 149-172.

Mella, I., Santos Rego, M. A., y Malheiro, X. M. (2015). Aprendizaje-servicio y rendimiento académico del alumnado universitario. Revista de Estudios e Investigación en Psicología y Educación, 12, 35-39. doi: 10.17979/ reipe.2015.0.12.569

Monge, A. (2003). Liderazgo educativo y retos de la educación superior en el siglo XXI: hacia la visión y la acción. Revista Electrónica Educare, 4, 63-75

Naval, C., y Arbués, E. (2016). El aprendizaje-servicio y la transición desde la educación superior al mundo del trabajo. En M. A. Santos Rego (Ed.), Sociedad del conocimiento. Aprendizaje e innovación en la universidad (pp. 219-239). Madrid: Biblioteca nueva.

Naval, C., y Arbués, E. (Eds.). (2018). Hacer la universidad en el espacio social. Pamplona: EUNSA.

Nieto, S. (Coord.) (2010). Principios, 
métodos y técnicas para la investigación educativa. Madrid: Dykinson.

Páez, M., y Puig, J. M. (2013). La reflexión en el aprendizaje-servicio. Revista Internacional de Educación para la Justicia Social, 2(2), 13-32.

Pérez-Serrano, M.G. (Coord.) (2011). Intervención sociocomunitaria. Madrid: UNED-Universidad Nacional de Educación a Distancia

Puig, J. M., Batlle, R., Bosch, C., y Palos, J. (2007). Aprendizaje servicio. Educar para la ciudadanía. Barcelona: Ministerio de Educación y Ciencia / Octaedro.

Salam, M., Awang, D. N., Abang, D. H., y Farooq, M. S. (2019). Technology integration in service-learning pedagogy: A holistic framework. Telematics and Informatics, 38, 257-273. doi: 10.1016/ j.tele.2019.02.002

Santos Rego, M. A. (Ed.). (2016). Sociedad del conocimiento. Aprendizaje e Innovación en la Universidad. Madrid: Biblioteca nueva.

Santos Rego, M. A. y Lorenzo, M. (Eds.) (2018). Guía para la institucionalización del aprendizaje-servicio en la universidad. Santiago de Compostela: Servizo de Publicacións e Intercambio de Científico.

Santos Rego, M. A., Mella, I., y Sotelino, A. (2020). Movilidad y TIC en aprendizaje-servicio: perspectivas para una sociedad global y tecnológica. Revista Iberoamericana de Educación a Distancia, 23(1), 67-84. doi: 10.5944/ ried.23.1.24180

Santos Rego, M. A., Sotelino, A., y Lorenzo, M. (2015). Aprendizaje-servicio y misión cívica de la universidad. Una propuesta de desarrollo. Barcelona: Octaedro.
Santos-Rego, M. A., Lorenzo, M., y Sotelino, A. (2018). Sostenibilidad e institucionalización cautelosa del aprendizajeservicio en la universidad. En C. Barroso (Coord.), Educación en la sociedad del conocimiento y desarrollo sostenible: XXXVII Seminario Interuniversitario de Teoría de la Educación (pp. 307-312). Tenerife: Universidad de La Laguna.

Sotelino, A., Mella, I., y Rodríguez Fernández, M. A. (2019). El papel de las entidades cívico-sociales en el aprendizaje-servicio. Sistematizando la participación del alumnado en el tercer sector. Teoría de la Educación, 31(2), 197-219. doi: 10.14201/teri.20156

Sotelino, A., Santos Rego, M. A., y Lorenzo, M. (2016). Aprender y servir en la universidad: una vía cívica al desarrollo educativo. Teoría de la educación, 28(2) 225-248. doi: 10.14201/teoredu 282225248

Välimaa, J., y Hoffman, D. (2008). Knowledge society discourse and higher education. Higher Education, 56, 265-285. doi: 10.1007/s10734-0089123-7

Vázquez Verdera, V. (2015) El aprendizaje-servicio: una estrategia para la formación de competencias en sostenibilidad. Foro de Educación, 19, 193-212.

Villa, A. (2006). El proceso de convergencia europeo y el papel del profesorado. Foro de Educación, 7-8, 103-117.

Waldner, L. S., McGorry, S. Y., y Widener, M. C. (2012). E-service-learning: The evolution of service-learning to engage a growing online student population. Journal of Higher Education Outreach and Engagement, 16(2), 123-150. 